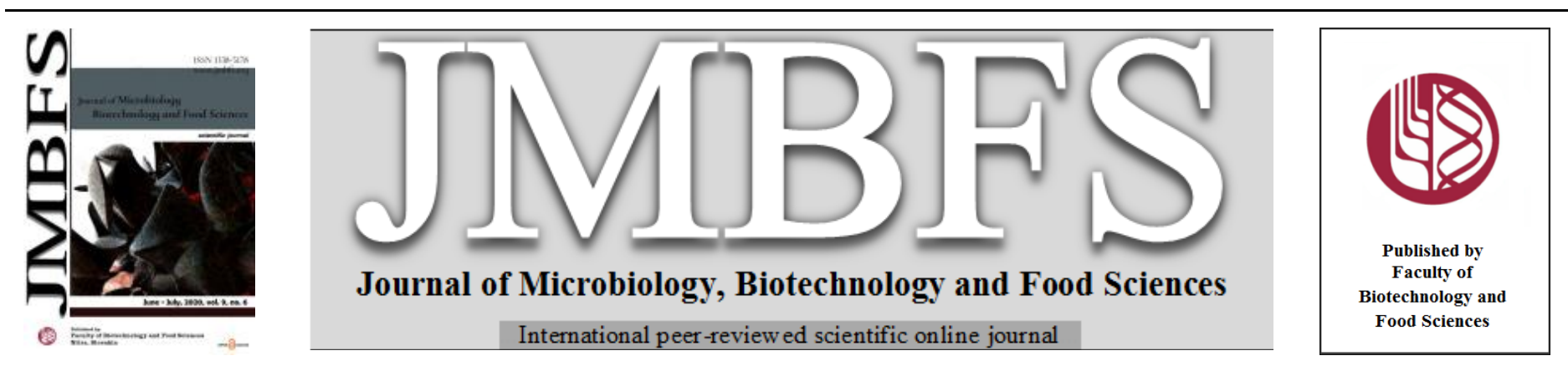

\title{
TESTING ALGORITHM IN CLOSTRIDIUM DIFFICILE INFECTION
}

\author{
Zuzana Stofkova ${ }^{*}$, Elena Novakova ${ }^{2}$, Vladimira Sadloňová ${ }^{3}$ \\ Address(es): MUDr. Ing. Zuzana Štofková, PhD. \\ ${ }^{1}$ Comenius University in Bratislava, Jessenius Faculty of Medicine in Martin, Institute of Microbiology and Immunology Mala Hora 4B, 03601 Martin, Slovakia, \\ +421904517772. \\ ${ }^{2}$ Comenius University in Bratislava, Jessenius Faculty of Medicine in Martin, Institute of Microbiology and Immunology, Mala Hora 4B, 03601 Martin, Slovakia. \\ ${ }^{3}$ Comenius University in Bratislava, Jessenius Faculty of Medicine in Martin, Institute of Microbiology and Immunology, Mala Hora 4B, 03601 Martin, Slovakia.
}

*Corresponding author: zuzana.stofkova@gmail.com

doi: 10.15414/jmbfs.2020.9.6.1211-1214

\section{ARTICLE INFO}

Received 7. 1. 2020

Revised 5. 2. 2020

Accepted 21. 2. 2020

Published 1. 6. 2020

Regular article

open $\partial_{\text {ACCESS }}$

\begin{abstract}
The aim of the study was to show that the multi-step algorithm contributes to fast and more accurate diagnostics and enables to start an early treatment of C.difficile infection (CDI). Materials for the study was 2,894 stool samples suspected of having CDI, were analyzed retrospectively. The biological samples from a nearby region were tested by different testing methods in Klinicka Biochemia laboratory in Zilina. The laboratory diagnosis was performed according to two- or three- step algorithm. The cultivation methods and identification of C.dificille strains by MALDI-TOF MS was performed. Most of the samples suspected of CDI could be assessed as negative within the applied two-step algorithm. In discordant results - samples with GDH positive and toxins A/B negative results were tested by further methods with higher sensitivity - ELISA test and the real-time PCR method in three-strp algorithm. The most positive results were from the departments of internal medicine and long-term-care patients over 60 years of age. Seasonality has shown a slight increase in positivity in the spring and autumn months, especially in 2015. The algorithm contributed to increasing the accuracy of the CDI suspect samples to reduce morbidity and mortality as well as the economic burden on CDI.
\end{abstract}

\section{INTRODUCTION}

Clostridium (Clostridioides) difficile is the most common pathogen of nosocomial and antibiotic-related diarrhea. Over the past decade, the number of infections caused by $C$. difficile has increased several times and ranks high in nosocomial diseases. (Khanna and Parda, 2010) During the European Centre for Disease Prevention and Control (ECDC) point prevalence survey of healthcare associated infections (HAI) and antimicrobial use in European acute care hospitals, $C$. difficile was the eighth most frequently found microorganism in HAI and the most common causative agent in gastrointestinal infections in health-care facilities. (ECDC, 2013) The incidence of CDI has been increasing worldwide partly due to the emergence of an epidemic strain (NAP1/027/BI) responsible for large outbreaks of severe CDI in the last few years. The hypervirulent strain referred as $C$. difficile BI / NAP1 / 027. (Jarcuska et al., 2015) The incidence of CDI in Slovakia grew up according to the study carried out by Novak et al. (Novak et al., 2018)

Accurate and fast diagnostics of CDI is essential optimal patient care and to prevent the spread of the infection. Colonization with $C$. difficile does not automatically lead to development of symptomatic CDI. (Gateau et. al., 2018) Colonization rates in healthy humans in the community range from $0.8 \%$ to $13 \%$ and are higher in long-term care facility residents. The interplay between the pathogenic virulence factors of the bacterium and the immune response of the host may in part explain how colonisation with $C$. difficile can result in wide spectrum of outcomes. (Solomon, 2013) The major virulence determinats of $C$. difficile strains are toxins A and B. Some $C$. difficile strains produce binary toxin (CDT), (Berry et al., 2017) which produced about $8 \%$ of the $C$. difficile strains. (Dudakova et al., 2011) The disease spectrum caused by $C$. difficile infection ranges from mild, self-limited, illness to a severe, life-threatening colitis. (Kachrimanidou and Malisiovas, 2009)

However, there are strains of $C$. difficile, which do not produce any of the toxins and are unable to induce the disease. (Debast et al., 2014) The diagnosis of CDI is based on a combination of symptoms confirmed by microbiological evidence of toxins produced by $C$. difficile or toxigenic strains of $C$. difficile in faeces, respectively colonoscopic evidence, or in the absence of other causes. (Murad $\boldsymbol{e t}$ al., 2016)
Studies have shown that rapid diagnosis has a positive impact on patient care, reducing delayed onset of patient isolation with CDI. (Gateau et. al., 2018) In order to manage $C$. difficile infections effectively, a rapid and accurate diagnostics is essential to guide treatment and to prevent transmission. (Crobach et al., 2016) According to ESCMID (European Society for Clinical Microbiology), the use of one standalone CDI test is not recommended due to the low positive predictive value at low CDI prevalence. (Gateau $\boldsymbol{e t .}$ al., 2018) Due to the different sensitivity of the different methods used, according to current recommendations, a combination of at least two different tests is preferred. (Burnham and Caroll, 2013) Differences in sensitivity and specificity, duration and cost of different test methods have resulted in laboratories using different test algorithms. Multiple laboratory tests detect different targets that determine the presence of free toxins or the presence of potential toxigenic strains. (Crobach $\boldsymbol{e t}$ al., 2016) The aim of the study was to show that the multi-step algorithm contributed to fast and more accurate diagnostics and enables to start an early treatment of $C$. difficile infection.

\section{MATERIAL AND METHODS}

The set of 2980 stool samples from patients suspected CDI patients submitted to Laboratory Klinicka Biochemia, Zilina. The testing for CDI was performed on physician's demand. A total number of 2894 samples suspected on CDI were tested by different testing methods and the results were processed in the study. The retrospective analysis was carried out from 2015-2017. The laboratory diagnosis was made according to the two- or three- step algorithm within biologic samples were tested with exoenzyme glutamate dehydrogenase (GDH) and toxin A/B EIA. The PCR (polymerase chain reaction) testing was applied as a third step in multi-step algorithm. All biologic samples received in the laboratory for diagnosis of CDI were tested in in- and outpatients. Other data as age, sex, type of department and comorbidities were analyzed. The stool samples suspected on CDI were tested by direct testing methods for detection of genes, enzymes and toxins in stool. The anaerobic culture was carried out simultaneously with other testing methods.

The laboratory tests detect different targets that determine the presence of free toxins or the presence of potential toxigenic C. difficile strains. The set of 2980 samples suspected on CDI were sent to the laboratory, 2894 samples met the 
testing criteria (amount of the biologic specimen, consistency, etc.). The patients' samples were tested by direct diagnostic methods for $C$. difficile using immunechromatography assay for the detection of Glutamate dehydrogenase enzyme (CERTEST Clostridium difficile GDH), toxins A or B (CERTEST Clostridium difficile Toxin A / B), immune-enzymatic methods ELISA (ProSpectT C.diffcile Toxin A / B Microplate assay) to determine toxins A and B. Selected biological samples were analyzed also by multiplex real-time PCR (GeneXpert, Cepheid). The anaerobic cultivation on taurocholate-cycloserine-cefoxitin-fructose agar was established to confirm positive $C$. difficile assays. The MALDI TOF method was used to identify $C$. difficile on species und subspecies level

The samples were directly cultured on selective media. The samples were inoculated into cycloserin-cefoxitin-fructose agar supplemented with taurocholate and incubated under anaerobic conditions at $37^{\circ} \mathrm{C}$ for 72 hours. C. difficile was identified by Matrix Assisted Laser Desorption/Ionisation Time-of-Flight Mass Spectrometry (MALDI-TOF MS) with the use of MALDI Biotyper v 3.0 system (Brucker Daltonics). Identification of anaerobic bacteria by MALDI-TOF method is fast, in comparison to conventional phenotypic techniques. C. difficile isolates were tested to vancomycin and metronidazole using E-test (BioMerieux) on blood agar (Oxoid). The minimum inhibitory concentration (MIC - minimal concentration that stops the growth of the bacterium) breakpoints for metronidazole and vancomycin were applied according to European Committee on Antimicrobial Susceptibility testing (EUCAST). The detection of toxins was carried out by multiplex „real-time“ PCR method (GeneXpert C. difficile Ep PCR Cepheid, Inc., CA) -enables the detection of the B toxin gene $(t c d B)$, the binary toxin genes( $c d t A a c d t B)$, and delecion of $t c d C$ gene on nucleotide 117 was carried out, which is suspecious for RT 027

\section{RESULTS AND DISCUSSION}

The group of 659 toxigenic strains of $C$. difficile samples $(22,77 \%)$ were positively tested of total samples (of 2980 samples) by different testing methods The samples were tested by glutamate dehydrogenase (GDH-EIA)/culture and enzyme immunoassay for $\mathrm{A} / \mathrm{B}$ toxins (immune-chromatography test). The number of stool samples were tested by at least two immune-enzymatic method (immune-chromatography for GDH and toxins A/B). $60 \%$ of stool samples suspected on CDI were tested with negative results by this algorithm. The highest proportion of positive samples tested by GDH-EIA test and test for toxins A/B EIA (two-step algorithm), according to the age group of the patients is shown in the figure 1 . The highest proportion of positive results were recorded from the age of 60 years in patients from all departments and from departments of internal medicine (INT) and long-term care department (LTC).

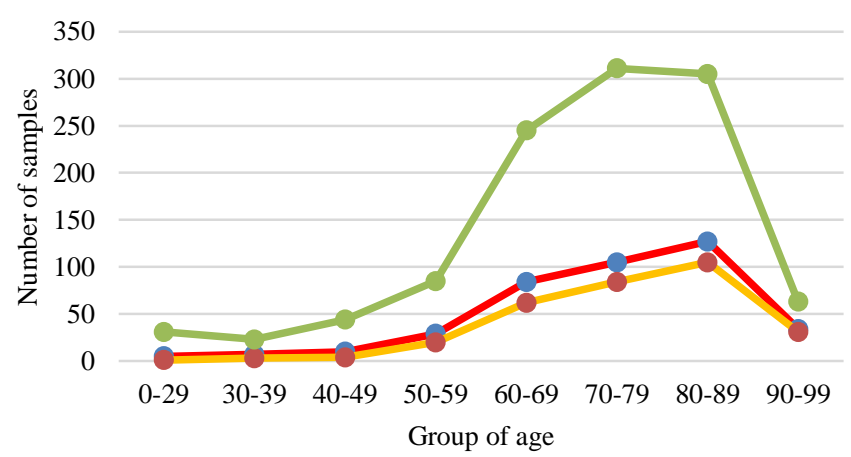

$\longrightarrow$ Positive $\longrightarrow$ Positive INT+LTC $\longrightarrow$ Negative

Figure 1 Number of positive samples in patients in 2-step algorithm in 2017 (GDH and toxins $\mathrm{A} / \mathrm{B}$ ) according to the age.

The median value for the age of patients with CDI positive samples was 76 years. $87 \%$ of all biologic samples were analysed from patients older than 60 years. The studies have confirmed that age is one of the risk factors for CDI. In total $47 \%$ of samples were from male patients and $53 \%$ were from female patients $77 \%$ of positively evaluated samples were from internal and long term wards patients where the proportion of females was $56 \%$, and of males $44 \%$.

The two-step algorithm including screening test for the detection of GDH and toxins A/B by immune-chromatography tests was enlarged from 2016 by ELISA testing method for detection of toxins $\mathrm{A} / \mathrm{B}$ and real-time PCR method for the detection of $\mathrm{B}$ toxin gene and genes for binary toxin. Almost $83 \%$ of samples suspected on CDI were tested as negative or positive within our two-step algorithm - testing by GDH/anaerobic culture and toxins A / B.

Discordant results in two-step algorithm - samples with GDH positive and toxins A/B negative results $-17 \%$ were tested by further testing methods with higher sensitivity - ELISA test and the real-time PCR method. On the demand of the physician, samples suspected on CDI were tested by PCR method in multi-step algorithm which could differentiate between toxigenic negative and toxigenic positive samples. The algorithm contributed to the accuracy, i.e. increased sensitivity and specificity of the testing methods, demonstrating the relevance of the multi-step testing procedure. The presence of co-morbidities predisposes these patients to more frequent hospitalizations. The chronic diseases are also significant risk factor for CDI. The most common chronic diseases in analysed patients with CDI were cardiovascular, pulmonary, renal diseases as well as oncological diseases.

Most $C$. difficile strains, $63 \%$ of tested samples were positive for toxins A / B $(A+B+)$. Almost in one third of GDH positive samples $-31 \%$ of samples were toxins $\mathrm{A} / \mathrm{B}$ negative (A- B-) - they were nontoxigenic strains or did not express the toxins (carriers), or the toxins $\mathrm{A} / \mathrm{B}$ were under the detection limit of the method in the biologic sample. The $C$. difficile strains were tested only on toxin $\mathrm{B}$ $(\mathrm{A}-\mathrm{B}+)$ in $5 \%$ of cases. The $C$. difficile strains were tested only on toxins $\mathrm{A} / \mathrm{B}$ $(\mathrm{A}+\mathrm{B}-)$ in $1 \%$ of cases. In our study most toxigenic $C$. difficile strains produced toxins $\mathrm{A}$ and $\mathrm{B}(\mathrm{A}+\mathrm{B}+)$ - in $91 \%$ (464 samples) of the samples. In 7\% (37 samples) samples - B toxin was detected (strains A-B +). C. difficile strains, which produce only toxin A (A + B-) - 2\% (10 samples), figure 2 . All positively tested samples were a rapid test, which separately examined toxin A and toxin B. All toxigenic strains confirmed for toxins in the next step by ELISA.

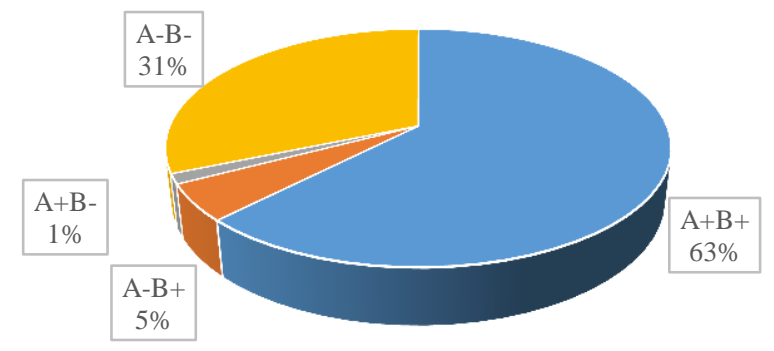

Figure 2 Samples tested positive for GDH and testing for toxins A/B in 2-step algorithm in 2017

During 2015, increased proportion of positive samples tested on CDI in individual months is displayed - figure 3 . The positive results were reported in the spring and autumn months during the year 2015.

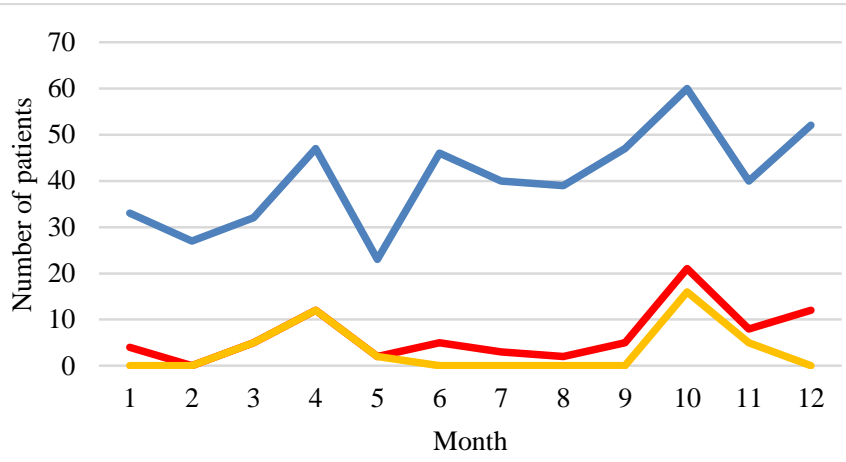

$\longrightarrow$ All positive 2 INT + LTC $\rightleftharpoons$ Positive + negative

Figure 3 Seasonality of tested samples in 2015

The proportion of positive tested samples on CDI in 2017 is displayed in figure 4 High proportion of positive results suspected on CDI was recorded during the whole year from internal department and LTC. In the spring and autumn period we observed a slight increase of positive samples. In the studied sample there was an average testing rate 1.5 per patient. 


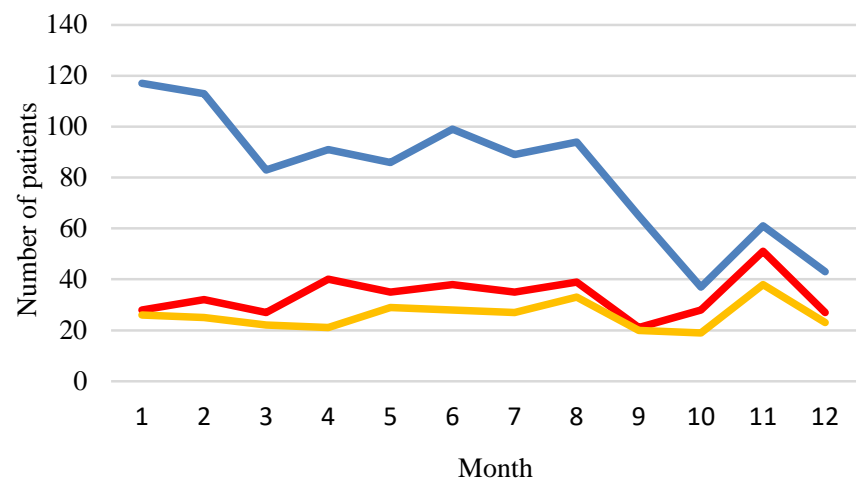

$\longrightarrow$ All positive $\longrightarrow$ INT + LTC $\longrightarrow$ Positive + negative

Figure 4 Seasonality of tested samples in 2017

There is still a suboptimal diagnosis of CDI prevalence in Europe. Early and accurate diagnosis is crucial for treatment management and prevention of disease spread. Correct diagnosis is a key factor for optimal treatment and prevention, but it remains a challenge. Differences in sensitivity and specificity, duration and cost of different test methods have resulted in laboratories using different testing algorithms. The optimal diagnostic approach to CDI diagnostics is still under discussion. Due to the different sensitivity of the different methods used, a combination of at least two different tests is preferred according to current recommendations. (Crobach $\boldsymbol{e t}$ al., 2016) Therefore, at least two-step algorithms are currently used to optimize CDI diagnosis. (Gateau $\boldsymbol{e t .}$ al., 2018)

The choice of examination method depends on the number of stools examined per day, the financial possibilities, etc. In the case of a positive result, a second test with a high positive predictive value is performed, i.e. a highly specific test, such as e.g. evidence of A / B toxins. If the second test is positive, it is most likely CDI. In a patient with a second negative toxin test, clinical signs should be evaluated. This may be a CDI infection (the amount of the toxin is not detected in an immune-enzymatic test, or it may be a carrier of a toxigenic strain

If the glutamate dehydrogenase (GDH) assay was performed as the first test in the order, then the real-time PCR assay can be performed as third step. (Crobach et al., 2016) Immuno-enzymatic methods based on the detection of toxin A and / or B are considered less sensitive to detection of toxigenic C. difficile. (Murad et al., 2016)

The samples that were tested negative for both GDH and toxin A/B can reliably be classified as non-CDI, while samples that test positive for both GDH and toxin A/B can be classified as CDI likely to be present. (Crobach et al., 2016) According to the Gilligan study, the 2-step algorithm increased the capture of toxigenic strains by $40 \%$ compared to the separate detection of toxins by immune-enzymatic methods. (Alfa and Sepehri, 2013) Within a two-step algorithm - GDH screening assay followed by toxin-EIA, $80-90 \%$ of the results may be labelled as negative or positive for up to approximately 4 hours. (Robotham and Wilcox, 2012)

The diagnosis of CDI was made according to the two- or three step algorithm (glutamate dehydrogenase EIA and EIA for C. difficile toxins A and B toxins and real-time PCR), and anaerobic culture. From 2015-2017 almost $83 \%$ samples could be evaluated negative or positive by the multi-step algorithm and the results could be sent on the day of testing. The other samples confirmed by ELISA test for the detection of toxins (higher sensitivity test) or real-time PCR method.

Samples that test negative for both GDH and toxin A/B can reliably be classified as non-CDI, while samples that test positive for both GDH and toxin A/B can be classified as CDI likely to be present. (Crobach $\boldsymbol{e t}$ al., 2016)

Interpretation of test results - if GDH (immune-enzymatic assay) or PCR positive and toxin ELISA are positive $(\mathrm{PPV}=91.4 \%)$ then $C$. difficile is most likely present. The result should be reported. If GDH is positive and toxin $\mathrm{A} / \mathrm{B}$ is negative, then $C$. difficile may be present, i. e. is potentially $C$. difficile carrier the results are not included in the mandatory reporting. If GDH (Immunoenzymatic) or PCR negative and toxin ELISA is negative (NPV $=98.9 \%)$ then it is very unlikely that $C$. difficile is present. (Robotham and Wilcox, 2012)

Multistep algorithm - including GDH, Toxin A/B, tcdB or tcdC gene comprises high accuracy and may help distinguish true $\mathrm{CDI}$ from $C$. difficile colonisation, when results are discordant (i.e. GDH positive and toxin negative), NAAT testing can confirm the correct diagnosis. (Bagdasarian and Rao, 2015)

In our studied samples the advantage of the algorithm (high sensitivity of GDH) and the increased proportion of positive samples (reduction of CDI false negative results) have shown that the introduction and implementation of the algorithm has led to an increased accuracy of the tested results. The PCR method could help to confirm the positive results (presence of toxigenic strains) in patients with positive symptomatology or to exclude the presence of toxigenic strains. In patients with positive symptomatology when other causes of diarrhoea were excluded the multi-step algorithm can help to guide the treatment of CDI above all in highly risk patients because of high NPV, and increased accuracy depending on high sensitivity and specificity of the PCR method.

GDH-EIA (the sensitivity is high, but specificity is lower because it produced also in other Clostridia or nontoxigenic strains of $C$. difficile).

In the studied sample the combination of testing methods with high sensitivity of enzyme immunoassay (GDH) and with sufficient specificity of enzyme immunoassay (e.g. immune-chromatography and ELISA) have shown that the implementation of the algorithm has led to an increased accuracy of the testing results. The PCR method could help to confirm the positive results (presence of toxigenic strains) in patients with positive clinical symptoms or to exclude the presence of toxigenic strains.

The diagnosis was made according to the two- or three step algorithm - glutamate dehydrogenase (EIA) and EIA for C. difficile toxins A and B toxins and real-time PCR and anaerobic culture. In our studied according to the two-step algorithm 83 $\%$ samples could be evaluated with positive or negative results, could be sent on the day of examination (4 hours). The other samples were confirmed by an ELISA test for the detection of toxins (higher sensitivity test) or by the real-time PCR method for the detection of toxin B gene and binary toxin genes.

If GDH (immune-enzymatic assay) is positive and toxin ELISA are positive, then $C$. difficile is most likely present. The result should be reported. If GDH is positive and toxin $\mathrm{A} / \mathrm{B}$ is negative, then $C$. difficile may be present, i. e. is potentially $C$. difficile carrier. If GDH (immune-enzymatic assay) and toxin by ELISA test is negative then it is very unlikely that $C$. difficile is present. (Robotham and Wilcox, 2012)

The advantage of an algorithm is that tests can be combined in such a way that the percentage of false-positive results can be decreased. (Crobach et al., 2016) The patients with negative second test for toxins should be clinically evaluated: they can be either truly infected with toxin level bellow the treshhold detection of the toxin assay or carriers of a toxigenic strain. (Gateau et. al., 2018)

In our algorithm $-31 \%$ of samples were GDH - positive and toxins A/B negative (A- B- strains of $C$. difficile ) - they were nontoxigenic strains or did not express the toxins (carriers), or the toxins $\mathrm{A} / \mathrm{B}$ were under the detection limit of the method in the biologic sample. These samples underwent further testing by ELISA test for toxins $\mathrm{A} / \mathrm{B}$ and by PCR method. According to studies most toxigenic strains of $C$. difficile produce both toxins $\mathrm{A}$ and $\mathrm{B}(\mathrm{A}+\mathrm{B}+)$. In our study $63 \%$ of tested samples were positive for toxins $\mathrm{A} / \mathrm{B}(\mathrm{A}+\mathrm{B}+)$. C. difficile positive only on $\mathrm{B}$ toxin were tested in $5 \%$ of cases. The $C$. difficile strains were tested only on toxins $\mathrm{A} / \mathrm{B}(\mathrm{A}+, \mathrm{B}-)$ in $1 \%$ of cases. We expect that these samples where the toxin $\mathrm{B}$ concentration was bellow the detection limit. The $C$. difficile strain which express only toxin A (A + B-) have been described only experimentally.

Most $C$. difficile strains, $63 \%$ of tested samples were positive for toxins A / B $(\mathrm{A}+\mathrm{B}+)$. Almost in one third of $\mathrm{GDH}$ positive samples The $C$. difficile strains were tested only on toxin B (A- B + ) in 5\% of cases. The $C$. difficile strains were tested only on toxins $\mathrm{A} / \mathrm{B}(\mathrm{A}+\mathrm{B}-)$ in $1 \%$ of cases. We expect that these samples where the toxin $\mathrm{B}$ concentration was bellow the detection limit. According to studies most $C$. difficile strains produce both toxins A and B, lower number of $C$. difficile expressed The $C$. difficile strains which express only toxin A (A + B-) have been described only experimentally.

It was documented that advanced age ( $\geq 65$ years), previous hospitalization, underlying disease, and prior use of antibiotics are important risk factors for CDI. (Peng et al., 2018) In many studies, age greater than 65 years was a risk factor for CDI. In our study $87 \%$ samples of patients were evaluated as positive in laboratory testing in the age group of patients with more than 65 years.

The risk factors for severe CDI are: age over 65 years, current treatment with broad spectrum antibiotics, long-term hospitalization, major underlying disease, etc. (Jarcuska et al., 2015)

In study carried out by Novak et. al $88,5 \%$ of patients samples positive on CDI were reported from internal wards. Analysis of the departments with the highest rates of occurence of CDI revealed that the highest proportion of positive CDI cases was on internal medicine ward and long term care wards (77\%), as well as ICU and surgery departments. These patients are at increased risk for CDI because of multiple comorbidities. The most common chronic diseases in analysed patients with CDI were cardiovascular diseases, pulmonary diseases, renal diseases as well as oncological diseases. In studied sample the positive results occured more often in female than in male samples what showed that women are slightly more affected by CDI $(53 \%)$ than men $(47 \%)$. The proportion of positive results in internal and longterm-care wards was in $56 \%$ by women and $44 \%$ by men.

The analysis of the seasonality of CDI among our patients gave similar conclusions as previous studies. Most previous studies reported that CDI is most common in the spring, and it is least common in the summer and fall. (FuruyaKanamori et al., 2015) When analysing the seasonality of CDI in 2015 the higher appearance in spring and lower appearance in summer, but a higher appearance occured in fall, in 2017 the laboratory findings did not show seasonal difference. All laboratory tests must be interpreted in relation to the clinical status of the patient and the risk factors for CDI. (Jarcuska et al., 2015)

The multistep algorithm - including GDH (EIA), toxins A/B, real-time PCR comprises high accuracy and may help distinguish true $\mathrm{CDI}$ from $C$. difficile colonisation, when results are discordant (i.e. GDH positive and toxin negative), NAAT testing can confirm the correct diagnosis. (Bagdasarian et al., 2015) 
The studies carried out by Krutova showed 5 samples with GDH-negative and toxin A/B-positive test results. It suggests that a third diagnostic step may be needed in order to confirm a GDH-negative toxin A/B-positive result. (Krutova et al., 2018)

EIA for toxins - immune-enzymatic methods based on the detection of toxin $\mathrm{A} / \mathrm{B}$ are considered less sensitive to the detection of toxigenic $C$. difficile than the PCR method. (Murad et al., 2016)

The rapid test (immunochromatography) showed in our sample slightly lower sensitivity as ELISA test. Studies have shown that toxin EIA are less sensitive than PCR and results are variable owing to toxin degradation associated with delayed sample testing. (Crobach,2016) The clinician should take into accoun that any diagnostic method, however sensitive, may be mistaken and be false negative. Taking into account the clinical symptoms of the patient and considering retesting for $C$. difficile. (Murad et al., 2016) A comprehensive assessment of a patient suspected of gastrointestinal infection requires the establishment of bacterial cultures, enzyme immunoassays, and possibly PCR screening. It is necessary to provide timely appropriate antibiotic therapy with early selection of patients who are at high risk and to perform a rapid selection of the most appropriate therapy. Predictive markers associated with poor prognosis of CDI (e.g. hemodialysis, intubation, etc.), laboratory predictive markers, such as differential blood count, acute phase protein levels, procalcitonin, lactate level, calprotectin, which are significant predictive factors as well as parameters that comprehensively assess water - mineral metabolism. (Jarcuska et al., 2015) In the context of CDI, there is a need for continuous awareness of the issue, as well as optimizing and evaluating diagnostic procedures and algorithms and early onset of the treatment, as well as barrier approach in the patient's managment, that can help reduce the incidence, morbidity and mortality of $C$. difficile infection. (Crobach,2016)

At the same time, the interpretation of results requires knowledge of the possible limits of specific diagnostic methods and experience in the clinical assessment of the results of specific diagnostic methods in clinical microbiology. Only patients with positive symptomatology should be tested. Due to the different sensitivity of the different methods used, according to current recommendations, a combination of at least two different tests is preferred. Reliable data is also crucial for monitoring CDI incidence over time and comparing individual healthcare facilities.

In the treatment and diagnosis of disease, laboratory findings should always be interpreted together with the patient's condition and other laboratory results, diagnostic methods and clinical condition of the patient, as well as the curren epidemiological situation. (Jarcuska et al., 2015) The algorithms currently recommended by the European Society for Clinical Microbiology and Infectious Diseases (ESCMID) include a combination of methods with high sensitivity and sufficient specificity. (Murad et al., 2016)

\section{CONCLUSION}

The aim of the study was to show the implementation of the multi-step algorithm in the routine diagnostics. The implemention fast and accurate laboratory test method enables starting an early treatment of $C$. difficile infection with decreasing of morbidity and mortality, preventing spread of infection and also economic burden. The advantage of an algorithm was to increase the sensitivity of the testing procedure and decrease the false-positive results (i.e. increase the specificity of testing methods).

The two- and three-step algorithmic approach for CDI testing was implemented in laboratory. In this approach, the samples were tested for exoenzyme glutamate dehydrogenase (GDH) and simultaneously immune-enzymatic assay or immunechromatography for toxins A/B was carried out. In the case of GDH positive samples and toxin negative samples in patients suspected of CDI, i.e. in discordant results, the ELISA test and real-time PCR were performed. In GDH positive results the culture was performed. By two-step algorithm - $83 \%$ of samples were evaluated positive or negative. The positivity rate in two-step algorithm was $22,77 \%$. The median value for the age of patients with CD positive samples was 76 years. $87 \%$ of all biologic samples were analysed from patients older than 60 years. The results from the two-step algorithm of testing could be sent to the physician on the day of testing, with the aim to confirm or to exclude the diagnosis of CDI and set up the early treatment. The clinica relevance of samples that are GDH-positive and toxin-negative and PCRpositive is an important issue. The patients may have CDI with low toxin levels that are not detectable with the EIA used. These results confirmed the importance of the pre-analytical phase (toxins are termolabile). The interpretation of laboratory findings have to be evaluated in relation to the clinical finding, other laboratory and radiological results of a particular patient and in relation to the current epidemiological situation. The analysis of the departments with the highest rates of occurence of CDI revealed that the highest proportion of positive $C$. difficile cases was on internal medicine department and long term care department, as well as ICU and surgery department. These patients are at increased risk of CDI because of present risk factors and multiple comorbidities.
Alfa, M. J. \& Sepehri, S. (2013). Combination of Culture, Antigen and Toxin Detection, and Cytotoxin Neutralization Assay for Optimal Clostridium difficile Diagnostic Testing. Canadian Journal of Infectious Diseases and Medical Microbiology, Volume 24, Issue 2, Pages 89-92. https://doi.org/10.1155/2013/934945

Bagdasarian, N., Rao, K. \& Malani, P. N. (2015). Diagnosis and Treatment of Clostridium difficile in Adults A Systematic Review. JAMA The Journal of the American Medical Association 313(4):398-408 https://doi.org/10.1001/jama.2014.17103

Berry, C. E., Davies, K. A., Owens, D. W. \& Wilcox, M. H. (2017). Is there a relationship between the presence of the binary toxin genes in Clostridium difficile strains and the severity of C. difficile infection (CDI)? Eur J Clin Microbiol Infect Dis. Dec;36 (12):2405-2415. https://doi.org/10.1007/s10096017-3075-8

Burnham, C.-A. D. \& Carroll, K. C. (2013). Diagnosis of Clostridium difficile Infection: an Ongoing Conundrum for Clinicians and for Clinical Laboratories Clinical Microbiology Reviews Vol. 26, Issue 3, 604-630. https://doi.org/10.1128/cmr.00016-13

Crobach, M. J. T., Planche, T., Eckert, C., Barbut, F., Terveer, E. M., Dekkers, O M., Wilcox, M. H. \& Kuijper, E. J. (2016). European Society of Clinical Microbiology and Infectious Diseases: update of the diagnostic guidance document for Clostridium difficile infection. Clinical Microbiology and Infection 22, 63-81. https://doi.org/10.1016/j.cmi.2016.03.010

Debast, M. P., Bauer, E. J. \& Kuijper, S. B. (2014). European Society of Clinical Microbiology and Infectious Diseases: update of the treatment guidance document for Clostridium difficile infection. Clinical Microbiology and Infection. Vol.20, Issue 2, 2014, pp. 1-26. Available: http://onlinelibrary.wiley.com/doi/10.1111/1469-0691.12418/full

Dudakova, L., Galova, Z. \& Melter, O. (2009). Genotyping of Clostridium difficile from patients with suspect infection cdad (Clostridium difficile associated disease). MendelNet'09 Agro conference, Brno 2009, p. 629-637.

European Centre for disease Prevention and Control (2013). Point prevelance survey at healthcare-associated infection and antimicrobial use in European acute care hospitals. Stockholm, ECDC

Furuya-Kanamori, L., McKenzie, S. J., Yakob, L., Clark, J., Paterson, D. L., Riley, T. V. \& Clemens, A. C. (2015). Clostridium difficile Infection Seasonality: Patterns across Hemispheres and Continents - A Systematic Review. PLOS ONE,

Vol. 10, Issue 3, e0120730. https://doi.org/10.1371/journal.pone.0120730

Gateau, C., Couturier, J., Coia, J. \& Barbut, F. (2018). How to: diagnose infection caused by Clostridium difficile. Clinical Microbiology and Infection 24, 463-468. https://doi.org/10.1016/j.cmi.2017.12.005

Jarcuska, P., Batovsky, M., Drgona, L. \& al. (2015). Odporúčaný postup diagnostiky a liečby kolitídy spôsobenej Clostridium difficile. Via practica; 1: 112. ISSN 1336-4790.

Kachrimanidou, M. \& Malisiovas, N. (2011). Clostridium difficile infection: comprehensive review. Crit Rev Microbiol. Aug;37(3):178-87. https://doi.org/10.3109/1040841x.2011.556598

Khanna, S. \& Pardi, D. S. (2010). The growing incidence and severity of Clostridium difficile infection in inpatient and outpatient settings. Journal Expert Review of Gastroenterology \& Hepatology, Volume 4, 2010, Issue 4, pp. 409 416. https://doi.org/10.1586/egh.10.48

Krutova, M., Wilcox, M. H. \& Kuijper, E. J. (2018). The pitfalls of laboratory diagnostics of Clostridium difficile infection, Clinical Microbiology and

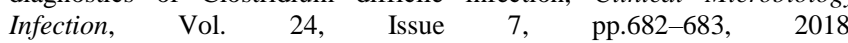
https://doi.org/10.1016/j.cmi.2018.02.026

Murad, Y. M., Perez, J., Ybazeta, G., Mavin, S., Lefebvre, S., Scott Weese, J., Rousseau, J., Diaz-Mitoma, F. \& Nokhbeh, R. (2016). False Negative Results in Clostridium difficile Testing. BMC Infect Dis. 16: 430 https://doi.org/10.1186/s12879-016-1741-6

Novak, M., Mazakova, I., Sadlonova, V., Cervenova, T. \& Hudeckova, H (2018). Appearance of Clostridium difficile infections in health care institutions in Slovakia and in the district of Martin. Acta Medica Martiniana, Vol.: 18 (2018), pp. 5-12. https://doi.org/10.2478/acm-2018-0007

Peng, Z., Ling, L., Stratton, C. V., Li, C., Polage, C. R., Wu, B. \& Tang, Y. W. (2018). Emerging Microbes \& Infections (2018) 7:15. Emerging Microbes \& Infections, Vol. 7, Issue 1, 1-13. https://doi.org/10.1038/s41426-017-0019-4

Robotham, J. \& Wilcox, M. (2012). Updated Guidance on the Diagnosis and Reporting of Clostridium Difficile. NHS, Advisory Committee on Antimicrobial Resistance and Healthcare Associated Infection (ARHAI), Retrieve from: https://www.gov.uk/ data/file/146808/dh_133016.pdf.pdf

Solomon, K. (2013). The host immune response to Clostridium difficile infection. Therapeutic Advances in Infectious Disease Vol. 1, Issue 1, 19-35. https://doi.org/10.1177/2049936112472173

\section{REFERENCES}

